\title{
New results of the $\mathrm{H}^{+} \rightarrow t b$ search using full Run-2 data with the ATLAS detector
}

\author{
Adrian Salvador Salas on behalf of the ATLAS Collaboration \\ Institut de Física d'Altes Energies, \\ Barcelona Institute of Science and Technology, \\ Barcelona, Spain \\ E-mail: asalvador@ifae.es
}

\begin{abstract}
A search for heavy charged Higgs bosons decaying to a pair of top and bottom quarks is presented. The analysis is performed using $139 \mathrm{fb}^{-1}$ of proton-proton collision data collected with the ATLAS detector at the Large Hadron Collider. The production of a heavy charged Higgs boson in association with a top and a bottom quarks, $p p \rightarrow t b H^{+} \rightarrow t b t b$, is explored in the $H^{+}$mass range from 200 to $2000 \mathrm{GeV}$ using multi-jet final states with one electron or muon. A massparameterised neural network is used to discriminate between signal and background events in four separate regions categorised according to the multiplicity of jets and jets originated from hadronisation of a bottom quark. No significant excess above the background-only hypothesis is observed and upper limits on the production cross-section times branching ratio of the charged Higgs boson as a function of its mass are derived. The results are interpreted in the context of the hMSSM and $\mathrm{M}_{\mathrm{h}}^{125}$ scenarios of the Minimal Supersymmetric Standard Model.
\end{abstract}

40th International Conference on High Energy physics - ICHEP2020

July 28 - August 6, 2020

Prague, Czech Republic (virtual meeting) 
The Standard Model (SM) of particle physics predicts the existence of only one neutral scalar boson, the Higgs boson, but many theories beyond the SM include an extended Higgs sector with at least five scalars, two of which are bosons with an electric charge, $H^{ \pm}$. Two-Higgs-doublet models (2HDM) feature the simplest extension of the Higgs sector, and the properties of the predicted charged Higgs boson ${ }^{1}$ depend on three parameters: its mass, the mixing angle of the neutral CPeven Higgs bosons, and the ratio of the vacuum expectation values of the two Higgs doublets, $\tan \beta$. In this document, a preliminary result of the search for $H^{+}$production in the $H^{+} \rightarrow t b$ decay mode with the full Run-2 dataset of $p p$ collisions taken at a centre-of-mass-energy of $13 \mathrm{TeV}$ by the ATLAS detector [1] at the Large Hadron Collider (LHC) is presented.

Events used in the analysis were recorded with single-lepton triggers and are required to have exactly one electron or muon with $p_{\mathrm{T}}>27 \mathrm{GeV}$ matched to the lepton trigger. A minimum of five jets are also required, with at least two originated from the hadronisation of a bottom quark, $b$-jets. With the isolation criteria applied both at the trigger and analysis levels, the background due to non-prompt leptons is expected to be negligible. In order to improve the sensitivity of the likelihood fit and constrain the systematic uncertainties, the selected events are classified into four separate regions according to the number of reconstructed jets ( $\mathrm{j}$ ) and $b$-jets (b): $5 \mathrm{j} 3 \mathrm{~b}, 5 \mathrm{j} \geq 4 \mathrm{~b}, \geq 6 \mathrm{j} 3 \mathrm{~b}$ and $\geq 6 \mathrm{j} \geq 4 \mathrm{~b}$. The main background is $t \bar{t}+$ jets, in particular $t \bar{t}+\geq 1 \mathrm{~b}$ in the most signal-sensitive regions. Data-based corrections are applied in the defined regions to improve the modelling of the high jet multiplicity events and the hardness of additional jet emissions. The correction factors are extracted from comparisons between data and simulation of the number of jets and the scalar sum of the $p_{\mathrm{T}}$, $H_{\mathrm{T}}$, of all jets and the lepton in the $\geq 5 \mathrm{j} 2 \mathrm{~b}$ region, where $t \bar{t}+\mathrm{jets}$ is the main background and the signal fraction is expected to be small. To further improve the modelling, the $t \bar{t}+$ jets background is subdivided into $t \bar{t}+\geq 1 \mathrm{~b}, t \bar{t}+\geq 1 \mathrm{c}$ and $t \bar{t}+$ light.

The search is performed in the $200-2000 \mathrm{GeV}$ mass interval and a mass-parameterised neural network (NN) [2] is used to enhance the separation between signal and background. The training is performed separately in each analysis region, with all the $H^{+}$mass samples used at the same time. The same training can be used to evaluate any $H^{+}$hypothesis in the defined mass range, as the value of the $\mathrm{H}^{+}$mass is an input parameter of the NN. The variables used in the NN are chosen to provide the best discrimination against background, with a kinematic discriminant, the $H_{\mathrm{T}}$ of all jets and the centrality ${ }^{2}$ being the most important ones. The binned NN score distributions corresponding to each analysis region are fitted simultaneously in a profile likelihood fit for each mass hypothesis. This technique is used to quantify the data agreement with background-only or background-plus-signal hypotheses, and to fit the best combination of signal and backgrounds yields together with the values of the other nuisance parameters that encode statistical and systematic uncertainties.

Data is found to be consistent with the background-only hypothesis and, for each mass hypothesis, exclusion limits are set at the $95 \%$ confidence level (CL) by using the $\mathrm{CL}_{\mathrm{S}}$ procedure [3] on $\sigma\left(p p \rightarrow t b H^{+}\right) \times \mathscr{B}\left(H^{+} \rightarrow t b\right)$, which range from $3.6 \mathrm{pb}$ at $200 \mathrm{GeV}$ to $0.035 \mathrm{pb}$ at $2000 \mathrm{GeV}$. Figure 1 shows the expected and observed exclusion limits as a function of the $\mathrm{H}^{+}$mass hypothesis as well as the limits set by the previous ATLAS search published in 2018 [4]. Figure 2 shows CL exclusion limits set on $\tan \beta$ versus $H^{+}$mass for the hMSSM and the $\mathrm{M}_{\mathrm{h}}^{125}$ scenarios.

${ }^{1}$ Charged Higgs bosons are denoted $H^{+}$for simplicity, with the charge-conjugate $H^{-}$implied.

${ }^{2}$ The centrality is defined as the $H_{\mathrm{T}}$ of all jets and leptons divided by the sum of their energies. 


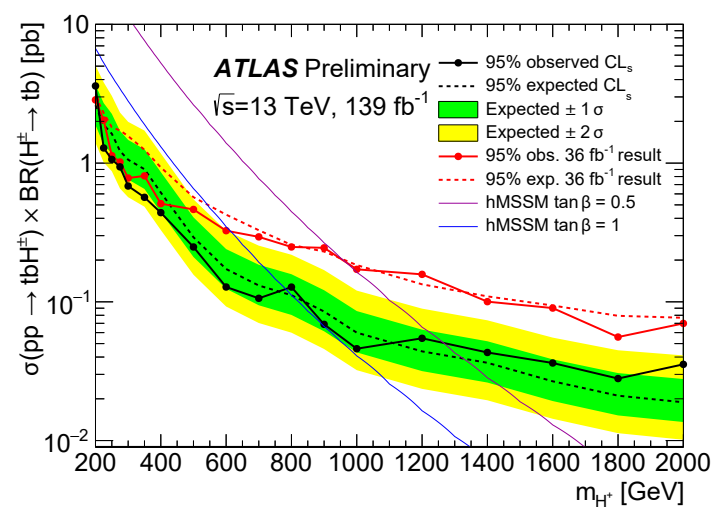

Figure 1: Expected and observed limits for the production of $p p \rightarrow t b H^{+} \rightarrow t b t b$ as a function of the $H^{+}$ mass. The bands surrounding the expected limit show the $68 \%$ and $95 \%$ confidence intervals. The red lines show the expected and observed 95\% CL exclusion limits obtained with the $36 \mathrm{fb}^{-1}$ data sample [5].
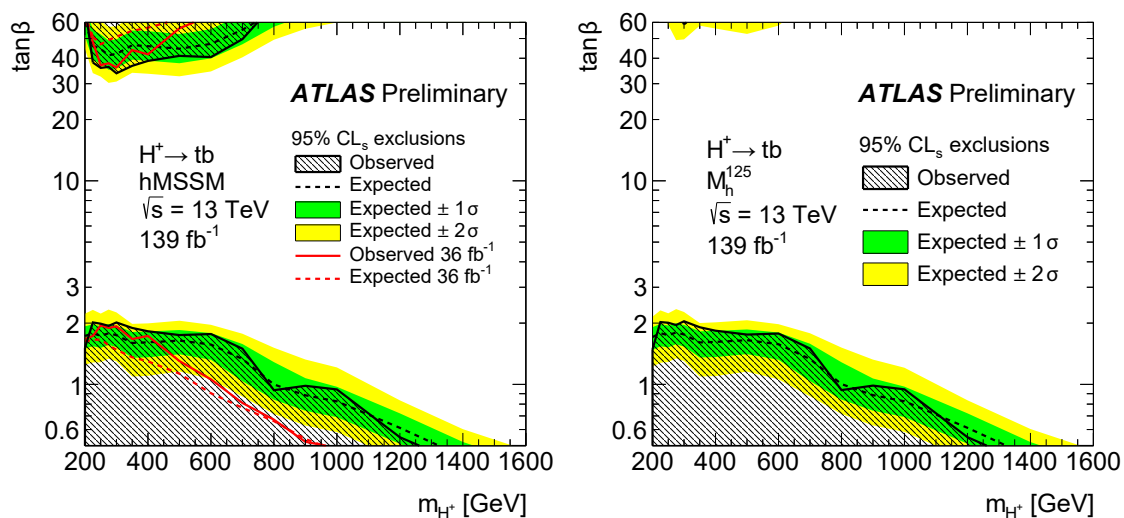

Figure 2: Expected and observed limits on $\tan \beta$ as a function of the $H^{+}$mass in the hMSSM (left) and the $M_{h}^{125}$ (right) scenarios [5].

\section{References}

[1] ATLAS Collaboration, The ATLAS experiment at the CERN large hadron collider, Journal of Instrumentation 3 (2008) S08003.

[2] P. Baldi, K. Cranmer, T. Faucett, P. Sadowski and D. Whiteson, Parameterized neural networks for high-energy physics, Eur. Phys. J. C 76 (2016) 235 [1601.07913].

[3] A.L. Read, Presentation of search results: The CL(s) technique, J. Phys. G 28 (2002) 2693.

[4] ATLAS Collaboration, Search for charged Higgs bosons decaying into top and bottom quarks at $\sqrt{s}=13 \mathrm{TeV}$ with the ATLAS detector, JHEP 11 (2018) 085 [1808.03599].

[5] ATLAS Collaboration, Search for charged Higgs bosons decaying into a top-quark and a bottom-quark at $\sqrt{s}=13 \mathrm{TeV}$ with the ATLAS detector, ATLAS-CONF-2020-039, Aug. 2020, https://cds.cern.ch/record/2727860. 